Full length article

\title{
Autophagic processes in Mytilus galloprovincialis hemocytes: Effects of Vibrio tapetis
}

\author{
Teresa Balbi ${ }^{\mathrm{a}}$, Katia Cortese ${ }^{\mathrm{b}}$, Caterina Ciacci ${ }^{\mathrm{c}}$, Grazia Bellese ${ }^{\mathrm{b}}$, Luigi Vezzulli ${ }^{\mathrm{a}}$, Carla Pruzzo ${ }^{\mathrm{a}}$, Laura Canesi ${ }^{\mathrm{a}}$ * \\ a Dept. of Earth, Environment and Life Sciences (DISTAV), University of Genoa, Italy \\ ${ }^{\mathrm{b}}$ Dept. of Experimental Medicine (DIMES), University of Genoa, Italy \\ ${ }^{\mathrm{c}}$ Dept. of Biomolecular Sciences (DISBM), University of Urbino, Italy
}

\section{ARTICLE INFO}

\section{Keywords:}

Bivalves

Hemocytes

Innate immunity

Lysosomes

Autophagy

Bacteria

Marine vibrios

\begin{abstract}
A B S T R A C T
Autophagy is a highly conserved and regulated catabolic process involved in maintaining cell homeostasis in response to different stressors. The autophagic machinery is also used as an innate immune mechanism against microbial infection. In invertebrates, that lack acquired immunity, autophagy may thus play a key role in the protection against potential pathogens. In aquatic molluscs, evidence has been provided for induction of autophagy by starvation and different environmental stressors; however, no information is available on autophagic pathways in the immune cells, the hemocytes.

In this work, the autophagic processes were investigated in the hemocytes of the marine bivalve, the mussel Mytilus galloprovincialis. The effects of classical inducers/inhibitors of mammalian autophagy were first tested. Rapamycin induced a decrease in lysosomal membrane stability-LMS that was prevented by the autophagy inhibitor Wortmannin. Increased MDC fluorescence and expression of LC3-II were also observed. Moreover, responses to in vitro challenge with the bivalve pathogen Vibrio tapetis were evaluated. Mussel hemocytes were unable to activate the immune response towards $V$. tapetis; however, bacterial challenge induced a moderate decrease in LMS, corresponding to lysosomal activation but no cytotoxicity; the effect was prevented by Wortmannin. TEM observations showed that $V$. tapetis resulted in rapid formation of autophagosomes and autolysosomes. Accordingly, increased LC3-II expression, decreased levels of phosphorylated mTor and of p62 were observed. The results represent the first evidence for autophagic processes in bivalve hemocytes in response to bacterial challenge, and underline the protective role of autophagy towards potential pathogenic vibrios.
\end{abstract}

\section{Introduction}

Macroautophagy (hereafter referred to as autophagy) is a highly regulated catabolic process by which cytoplasmic components, including macromolecules and organelles, are sequestered into double-membrane vesicles, the autophagosomes, and then delivered to lysosomes for degradation [1]. Autophagy is involved in maintaining cell homeostasis in response to extra- or intracellular stress signals such as starvation, growth factor deprivation, endoplasmic reticulum stress, pathogen infection [2,3]. A number of key genes and signaling pathways involved in autophagy have been identified, including Phosphatidylinositol 3-kinase (PI3K), the mechanistic Target of rapamycin (mTor), and different autophagy related proteins (ATGs) that are highly conserved among eukaryotes [2]. The autophagic machinery is also used to remove invading intracellular pathogens and to direct them toward lysosomal degradation, and it is therefore considered an innate immune mechanism against microbial infection (xenophagy) [4]. In this light, in invertebrates, that lack acquired immunity, autophagy may play a key role in the protection against potential pathogens.

Although the role of autophagy in different physiological processes has been described in the model invertebrates Drosophila melanogaster and Caenorabditis elegans [5,6], little is known in other invertebrate groups. Among aquatic invertebrates, molluscs have been shown to provide useful models for studying the autophagic function. Autophagy has been mainly demonstrated by different types of lysosomal reactions in the cells of the hepatopancreas in response to a variety of environmental stressors (starvation, salinity change, hyperthermia, hypoxia, pollutant-induced stress) [7-9]. More recently, biochemical and molecular evidence has been provided for the in vivo induction of autophagic processes, including expression of ATG genes, in marine invertebrates,

\footnotetext{
* Corresponding author. Dept. of Earth, Environment and Life Sciences (DISTAV), University of Genoa, Corso Europa 26, 16132 Genoa, Italy.

Email address: Laura.Canesi@unige.it (L. Canesi)
} 
such as the pacific oyster Crassostrea gigas [10] and the crab Eriocheir sinensis [11], suggesting a protective role against infection.

In the marine bivalve, the mussel Mytilus spp., immune cells (hemocytes) are mainly represented by granular cells, endowed with an extremely developed lysosomal vacuolar system $[12,13]$. Despite several pathogens (different viruses and vibrio strains) have been associated with mortality outbreaks of bivalve species, Mytilus is considered particularly resistant to infection, due to the capacity to mount an efficient immune response against a wide range of microorganisms (reviewed in Ref. [13]). In this light, Mytilus hemocytes provided a suitable in vitro model for studying the signaling components involved in the activation of the immune response to bacterial challenge [14]. The results so far obtained underlined the role of the lysosomal system and of different kinase-mediated intracellular pathways in the response to different vibrio species and strains [13 and references quoted therein].

In this work, the autophagic processes were investigated in the hemocytes of the Mediterranean mussel M. galloprovincialis. The effects of autophagy inducers/inhibitors were first evaluated. Moreover, the effects of in vitro challenge with the bivalve pathogen Vibrio tapetis were investigated. Different immune responses were measured, such as extracellular oxyradical production and bactericidal activity. Lysosomal membrane stability (LMS) was determined as the most sensitive lysosomal response to cellular stress [9]. Other lysosomal and autophagic markers were evaluated by Confocal laser scanning microscopy (CLSM), transmission electron microscopy (TEM), electrophoresis and Western blotting.

\section{Materials and methods}

\subsection{Animals and hemolymph collection}

Mussels (Mytilus galloprovincialis Lam), 4-5 cm long, were purchased from an aquaculture farm (Arborea-OR, Italy) in October 2016 and kept for 1 day in static tanks containing aerated artificial sea water (ASW), salinity $36 \mathrm{ppt}\left(1 \mathrm{~L} \mathrm{mussel}^{-1}\right)$ at $18{ }^{\circ} \mathrm{C}$. Hemolymph was extracted from the posterior adductor muscle using a sterile $1 \mathrm{~mL}$ syringe with an $18 \mathrm{G} 1 / 2^{\prime \prime}$ needle. With the needle removed, hemolymph was filtered through a sterile gauze and pooled in $50 \mathrm{~mL}$ Falcon tubes at $18^{\circ} \mathrm{C}$. Hemolymph serum was obtained by centrifugation of whole hemolymph at $100 \times \mathrm{g}$ for $10 \mathrm{~min}$, and the supernatant was sterilized through a $0.22 \mu \mathrm{m}$-pore filter. Hemocyte monolayers were prepared as previously described $[15,16]$.

\subsection{Determination of lysosomal membrane stability-LMS}

Lysosomal membrane stability-LMS in hemocyte monolayers was evaluated by the NRRT assay as previously described $[9,15,16]$. The endpoint of the assay was defined as the time at which $50 \%$ of the cells showed sign of lysosomal leaking (the cytosol becoming red and the cells rounded). In experiments with autophagy inducers/inhibitors, cells were pretreated for $60 \mathrm{~min}$ with different concentrations of Rapamycin, and 20 min with $0.1 \mu \mathrm{M}$ Wortmannin (for PI3-kinase) suitably diluted in serum from $10 \mathrm{mM}$ stock solutions in DMSO (dymethyl sulphoxide) [15].

\subsection{Confocal laser scanning microscopy (CLSM)}

Hemocytes treated with Rapamycin as described above were loaded with Acridine orange-AO $(0.25 \mu \mathrm{M}$ for $15 \mathrm{~min})$ and Tetramethylrhodamine, ethyl ester-TMRE ( $40 \mathrm{nM}$ for $10 \mathrm{~min}$ ) for determination of lysosomal staining and mitochondrial membrane potential respectively. Cells were also stained with the acidotropic dye Monodansylcadaverine-MDC (50 $\mu \mathrm{M}$ for $30 \mathrm{~min}$ ). Fluorescence of AO (ex: $502 \mathrm{~nm}$, em: 490
- >560 nm), TMRE (ex: 488 nm, em: 580) and MDC (ex: 380 nm, em: $530 \mathrm{~nm}$ ) was detected using a Leica TCS SP5 confocal setup mounted on a Leica DMI 6000 CS inverted microscope (Leica Microsystems, Heidelberg, Germany) using $63 \times 1.4$ oil objective (HCX PL APO 63.01 .40 OIL UV). Images were analyzed by the Leica Application Suite Advanced Fluorescence (LASAF) and ImageJ Software (Wayne Rasband, Bethesda, MA).

2.4. Bacterial cultures and in vitro challenge of Mytilus hemocytes with $V$. tapetis

Vibrio tapetis LP2 were kindly provided by Dr. Christine Paillard (Institut Universitaire European de la Mer French National Centre for Scientific Research, IUEM, Paris, France). Bacterial cells were cultured in Zobell Marine Broth 2216 (Difco Laboratories) at $20^{\circ} \mathrm{C}$ under static conditions; after overnight growth, cells were harvested by centrifugation ( $4500 \times g, 15 \mathrm{~min}$ ), washed three times with artificial seawater (ASW), and resuspended to an $\mathrm{A}_{600}=1$ (about $10^{8} \mathrm{CFU} / \mathrm{mL}$ ). Marine Agar (Conda Lab, Spain) was also used throughout the experiments. Hemocyte monolayers were incubated at $18^{\circ} \mathrm{C}$ with suspensions of $\mathrm{V}$. tapetis suitably diluted in hemolymph serum at a $10^{7}$ or $10^{6} \mathrm{CFU} / \mathrm{mL}$, for different periods of times, depending on the endpoint measured. Untreated hemocyte samples in serum were run in parallel. All experiments were performed in triplicate.

\subsection{Effects of $V$. tapetis on hemocyte functional parameters}

All assays were carried out as previously described $[15,16]$. Hemocyte monolayers were incubated with suspensions of $V$. tapetis $\left(10^{7} \mathrm{CFU} / \mathrm{mL}\right)$ in hemolymph serum for $30 \mathrm{~min}$ and then tested for extracellular generation of reactive oxygen species (ROS) by the reduction of cytochrome c. Samples were read at $550 \mathrm{~nm}$ and the results expressed as changes in OD per mg protein. Control hemocytes were run in parallel. Lysozyme release and Nitric oxide production were also evaluated as described in Refs. $[15,16]$. Protein content was determined according to the bicinchoninic acid (BCA) method using bovine serum albumin (BSA) as a standard. Bactericidal activity was evaluated in hemocyte monolayers incubated with $V$. tapetis at $18^{\circ} \mathrm{C}$ for different periods of time (30-90 $\mathrm{min})$. Immediately after the inoculum $(\mathrm{T}=0)$ and at different times of incubation at $18{ }^{\circ} \mathrm{C}$, supernatants were collected and hemocytes were lysed by adding $0.5 \mathrm{~mL}$ of filter sterilized ASW supplemented with $0.05 \%$ Triton $\mathrm{x}-100$ and by $10 \mathrm{~s}$ agitation. Supernatants and lysates were pooled and tenfold serial diluted in ASW. Aliquots $(100 \mu \mathrm{L})$ of the diluted samples were plated onto Marine Agar. After overnight incubation at $20^{\circ} \mathrm{C}$ the number of colony-forming units (CFU) per hemocyte monolayer (representing live, culturable bacteria) was determined. The number of CFU in control hemocytes never exceeded $0.1 \%$ of those enumerated in experimental samples.

LMS was evaluated by the NRRT assay in control hemocytes and hemocytes incubated for 30 min with $V$. tapetis $\left(10^{7} \mathrm{CFU} / \mathrm{mL}\right)$. Determination of LMS was also carried out in hemocytes pretreated with Wortmannin as described above and then challenged with $V$. tapetis.

\subsection{Transmission electron microscopy (TEM)}

TEM of mussel hemocytes was carried out as previously described [15]. Hemocyte monolayers were seeded on glass chamber slides for $20 \mathrm{~min}$ at $18^{\circ} \mathrm{C}$ (Lab-Tek, Nunc, 177380), and incubated with $V$. tapetis for 5, 15 and $30 \mathrm{~min}$. Samples were washed out with $0.1 \mathrm{M}$ cacodylate buffer in ASW and fixed in $0.1 \mathrm{M}$ cacodylate buffer in ASW containing $2.5 \%$ glutaraldehyde in ASW, for $1 \mathrm{~h}$ at room temperature. The cells were postfixed in $1 \%$ osmium tetroxide in ASW for 10 min and $1 \%$ 
uranyl acetate in ASW for $1 \mathrm{~h}$. Subsequently, samples were dehydrated through a graded ethanol series and embedded in epoxy resin (PolyBed; Polysciences, Inc., Warrington, PA) overnight at $60^{\circ} \mathrm{C}$. About 50 cells per sample were observed by F20 Tecnai electron microscope (Philips, Eindhoven, The Netherlands), and representative images were taken with an Eagle CCD camera and iTEM software and processed with Adobe Photoshop CS2.3.2.

\subsection{Electrophoresis and western blotting}

Hemocyte monolayers were incubated with bacterial suspensions in hemolymph serum $\left(10^{7} \mathrm{CFU} / \mathrm{mL}\right)$ at $18^{\circ} \mathrm{C}$ for 5,15 and $30 \mathrm{~min}$. Control hemocytes were incubated in the same volume of serum. Supernatants from each culture dish were discarded and the hemocytes were lysed with $0.1 \mathrm{vol}$ of ice-cold lysis buffer $(50 \mathrm{mM}$ Tris- $\mathrm{HCl} \mathrm{pH}$ $7.8,0.25 \mathrm{M}$ sucrose, $1 \% \mathrm{SDS}, 2 \mathrm{mM}$ sodium orthovanadate, $0.1 \%$ Nonidet-P40, $5 \mathrm{mM}$ M DTT) with protease inhibitors cocktail and phosphatase inhibitors cocktail (Roche, Basel, Switzerland). Samples were boiled for $4 \mathrm{~min}$ and then centrifuged for $20 \mathrm{~min}$ at $14,000 \times \mathrm{g}$ to remove insoluble debris.

Levels of target proteins were determined by SDS-PAGE and Western blotting using specific antibodies as previously described $[14,16]$. Supernatants were mixed 1:1 (v:v) with sample buffer $(0.5 \mathrm{M}$ Tris- $\mathrm{HCl} \mathrm{pH}$ 6.8, 2\% SDS, 10\% glycerol, 4\% 2-mercaptoethanol, 0.05\% Bromophenol blue) and samples (normalized to $15 \mu \mathrm{g}$ of protein before loading) were resolved by SDS-polyacrylamide gel electrophoresis on Bolt 4-12\% Bis-Tris Plus (Invitrogen). Pre-stained molecular mass markers SeeBlue Plus2 (Novex by Life technologies) were run on adjacent lanes. The gels were electro-blotted and stained with Coomassie blue according to [17]. Blots were probed with primary antibodies: rabbit monoclonal [EPR427(N)] to mTor (phospho S2481) (1:1000), rabbit polyclonal anti-LC3 (Sigma-Aldrich, L8918) antibodies (1:500), Anti-p62 SQSTM1 monoclonal antibody (M01), clone 2C11 (Abnova H00008878-M0), rabbit polyclonal anti-actin antibody (Sigma-Aldrich, A5060) (1:500). Horseradish peroxidase-conjugated goat anti-rabbit IgG (Invitrogen G21234) and anti-mouse IgG (Invitrogen, G21040) (1:3000) were utilized as secondary antibodies. Membranes were stripped and re-probed with rabbit polyclonal anti-mTor antibody ab2732 (www.abcam.com) (1:2000). Immune complexes were visualized using an enhanced chemiluminescence Western blotting analysis system (PURECL-Chemiluminescence Substrate Vilber) following the manufacturer's specifications. Western blot films were digitized (Chemidoc-Biorad) and band optical densities (arbitrary units) were quantified using a computerized imaging system (QuantityOne).

\subsection{Data analysis}

The results are the mean \pm SD of at least 4 experiments and analyses, unless otherwise indicated, performed in triplicate. Statistical analysis was performed by ANOVA followed by Tukey's post hoc test or by Mann-Whitney $U$ test using the GraphPad Instat software.

\section{Results}

\subsection{Effects of autophagy activators/inhibitors on Mytilus hemocytes}

We first tested the possible effects of classical inducers/inhibitors of mammalian autophagy on hemocyte LMS, as a simple and sensitive quantitative marker of lysosomal reactions, evaluated by the Neutral Red Retention time assay [7-9,18]. Exposure of Mytilus hemocytes to Rapamycin (the mTor inhibitor which induces autophagy) for $60 \mathrm{~min}$ induced a dose-dependent decrease in LMS in $0.1-1 \mu \mathrm{M}$ range (Fig. 1).

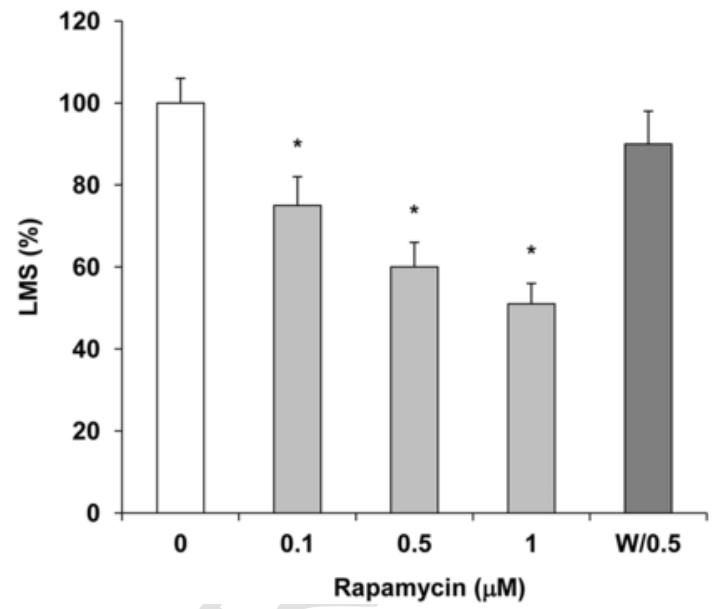

Fig. 1. Effects of autophagy activators/inhibitors on lysosomal membrane stability (LMS) of Mytilus hemocytes. Hemocyte monolayers were treated with different concentrations $(0.1-0.5-1 \mu \mathrm{M})$ of Rapamycin for $60 \mathrm{~min}$ and LMS was evaluated by the NRRT assay. The effect of cell pretreatment $(20 \mathrm{~min})$ with $0.1 \mu \mathrm{M}$ Wortmannin $(\mathrm{W})$ on cells exposed to $0.5 \mu \mathrm{M}$ Rapamycin (W/0.5) are also reported. Data, expressed as percent values with respect to controls and representing the mean \pm SD of 4 experiments in triplicate, were analysed by ANOVA followed by Tukey's post hoc test. ${ }^{*} \mathrm{P} \leq 0.01$.

In contrast, no significant effects were observed with another autophagy inducer, carbamazepine, at concentrations up to $100 \mu \mathrm{M}$ (not shown). The effect of Rapamycin on hemocyte LMS was prevented by cell pre-treatment with Wortmannin, a potent inhibitor of PI-3Kinases, that play a key role in autophagosome formation [2].

CLSM showed that Rapamycin also increased fluorescence of acridine orange (AO), corresponding to lysosomal enlargement and acidification (Fig. 2A-B), and of Tetramethyl rhodamine ethyl ester (TMRE), indicating decreased mitochondrial membrane potential $\left(\Delta_{\Psi}\right)$ (Fig. 2C-D). Monodansylcadaverine (MDC) was also utilized as an in vivo marker for acidic compartments (acidic endosomes, lysosomes, and late-stage autophagosomes), whose labeling is upregulated under conditions that increase autophagy [19]. MDC fluorescence was barely detectable in control cells, and it was dramatically increased by Rapamycin exposure (Fig. 3A-B). Moreover, expression of MAP1LC3/LC3 (microtubule-associated protein 1 light chain 3), frequently used as an autophagosome marker, was evaluated. In hemocyte cytosolic fractions, two immunoreactive bands were detected of the MW of 16 and 14 Kda, corresponding to LC3-I and LC3-II, respectively. LC3 immunoreactivity was generally low, as previously shown in oyster mantle and sea urchin embryos for LC3-1 in particular [10,20]. Rapamycin significantly increased the expression of the activated, lipidated form LC3-II, evaluated by LC3-II/LC3-I ratio (Fig. 3C) and, to a similar extent, the LC3-II/actin ratio (not shown).

\subsection{Functional responses of mussel hemocytes to challenge with $V$. tapetis}

Hemocytes were challenged in vitro with $V$. tapetis $\left(10^{7} \mathrm{CFU} / \mathrm{mL}\right)$ and different functional parameters were evaluated in the same conditions previously utilized for other vibrio species and strains [14-16]. $V$. tapetis did not induce extracellular lysozyme release and NO production (not shown); in addition, bacterial challenge did not activate the oxidative burst, but even reduced the basal production of extracellular ROS (Fig. 4A). The capacity of mussel hemocytes to kill V. tapetis was evaluated in a bactericidal assay that quantifies the number of live, cultivable bacteria at different times of incubation. No bactericidal activity was observed, even at lower bacterial concentrations $\left(10^{6} \mathrm{CFU} / \mathrm{mL}\right)$ (Fig. 4B). On the other hand, incubation with $V$. tapetis induced a moderate decrease in LMS $(-30 \%$; $\mathrm{P} \leq 0.05)$ (Fig. $4 \mathrm{C}$ ), indicating activa- 

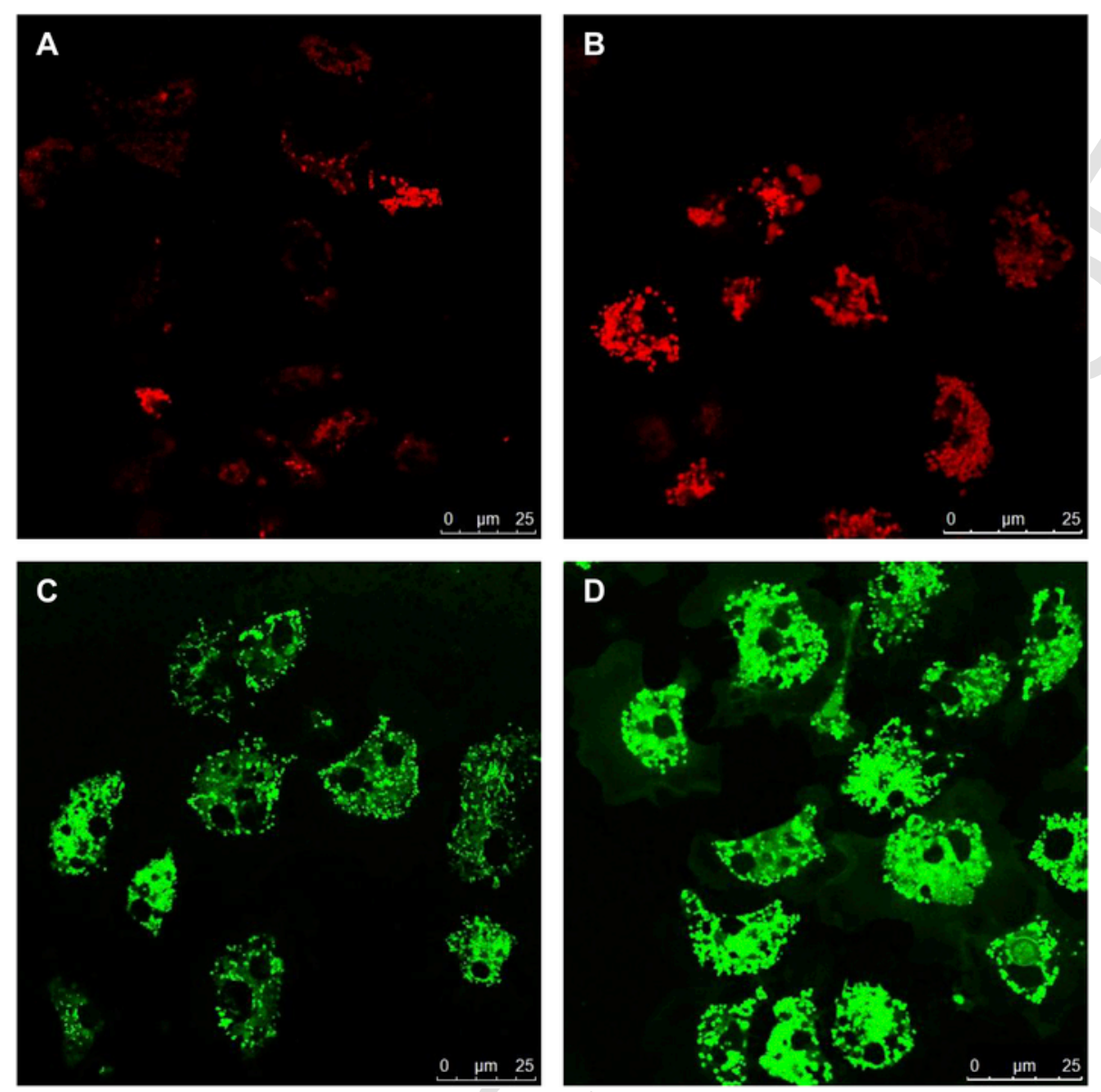

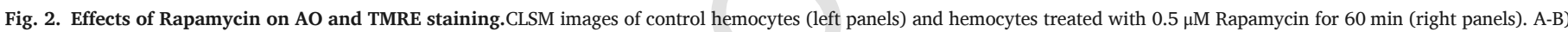

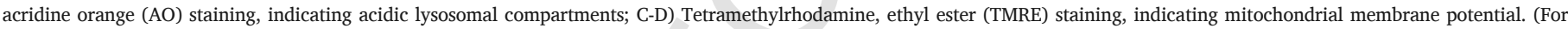
interpretation of the references to colour in this figure legend, the reader is referred to the Web version of this article.)
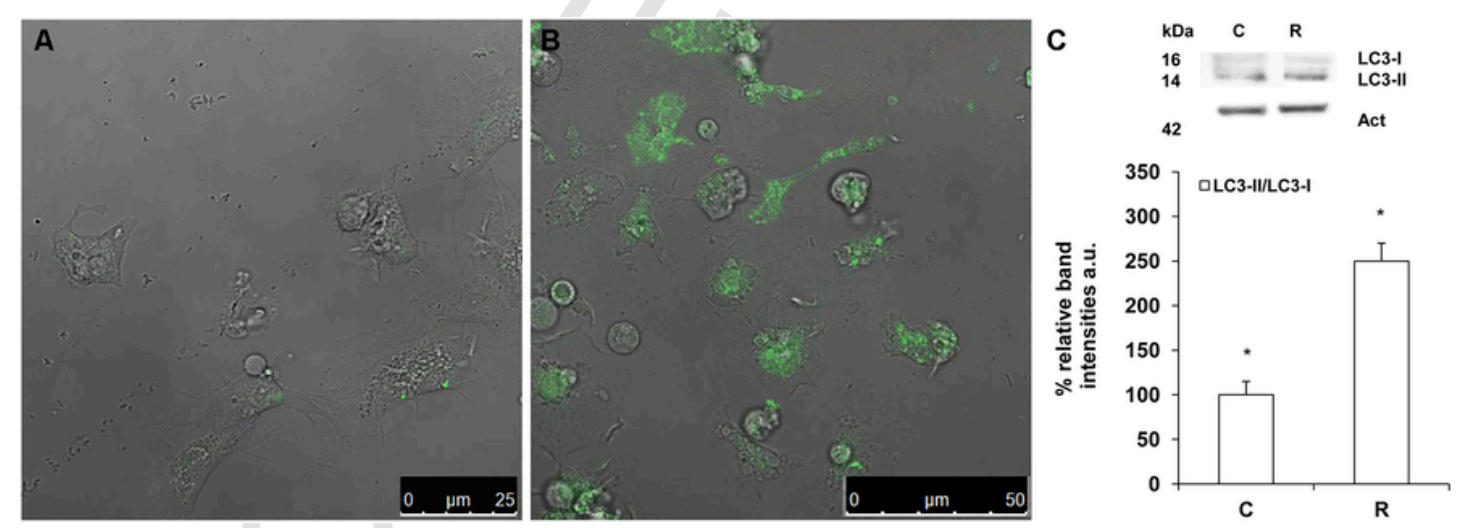

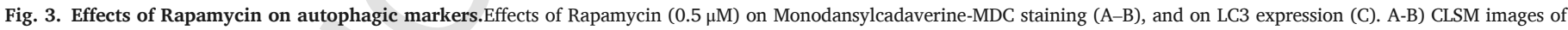

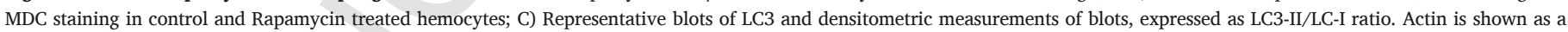
loading control. Data from 4 independent experiments are presented as mean \pm SD. ${ }^{*} \mathrm{P} \leq 0.05$, Mann-Whitney $U$ test.

tion of the lysosomal system in the absence of cytotoxicity [18]. Cell pre-treatment with Wortmannin prevented the lysosomal destabilization induced by $V$. tapetis.

\subsection{Transmission electron microscopy-TEM}

Hemocytes incubated with $V$. tapetis for different times (5, 15 and $30 \mathrm{~min}$ ) were observed by TEM and the results are reported in Fig. 5. Fig. 5A shows a representative image of $V$. tapetis before addition to the hemocytes, After incubation, only few cells showed internalization of individual and intact bacteria within single membrane vesicles (Fig. $5 B, C, D)$. In some cases, the vesicles showed an outer layer of granular material and a clear inner zone around bacterial cells (Fig. 5E-F), this latter suggesting that these bacteria maybe actively secreting toxins. The appearance of bacteria-containing vacuoles was independent of time of exposure; similarly, the morphological integrity of internalized vibrios did not change over time, indicating no bacterial degradation. 

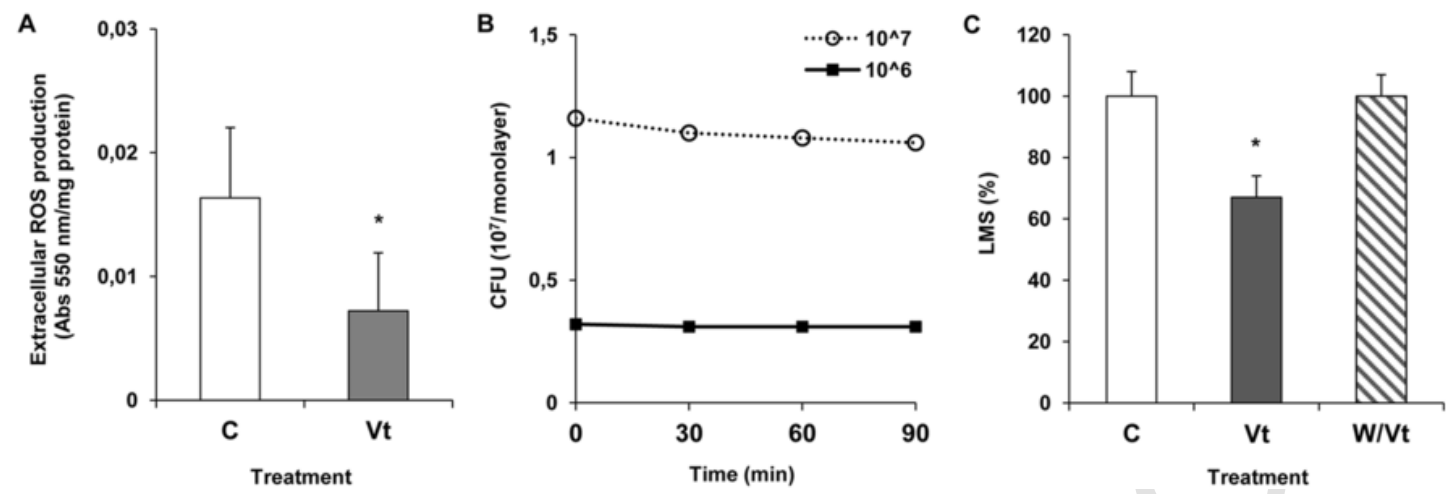

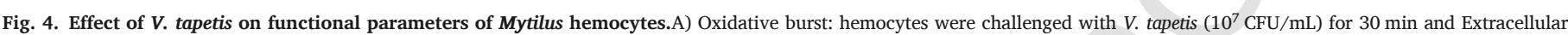

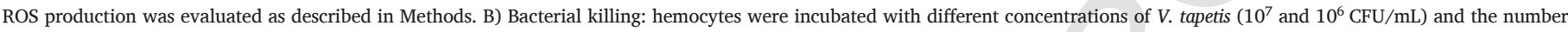

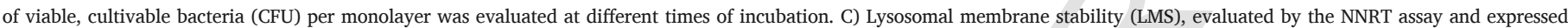

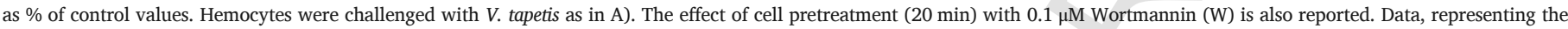
mean \pm SD of 4 experiments in triplicate, were analysed by ANOVA followed by Tukey's post hoc test. *P $\leq 0.01$.
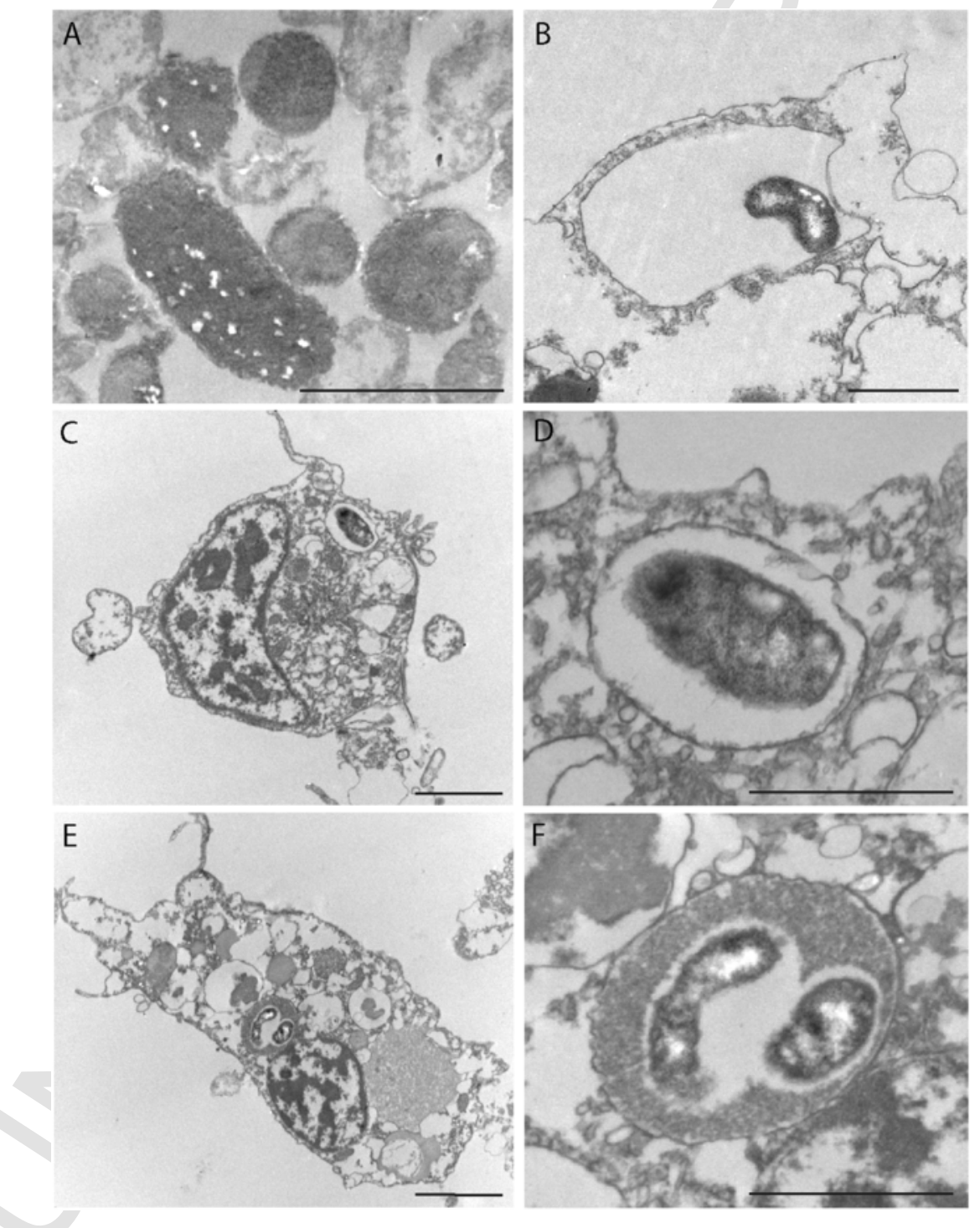

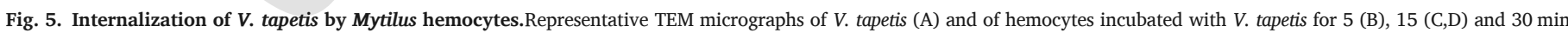

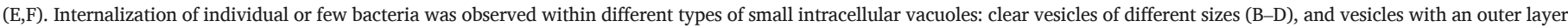

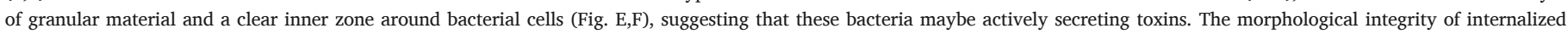
vibrios did not change over time, indicating no bacterial degradation. Scale bars: $2 \mu \mathrm{m}$. 
As previously shown by TEM [15], M. galloprovincialis hemocytes are mainly represented by granular cells, characterized by the presence of a rich lysosomal vacuolar system in the form several granules of different sizes (hundreds nms) and electron densities (Fig. S1). Incubation with $V$. tapetis induced remarkable changes in their intracellular structure (Fig. 6). As soon as 5 min post incubation (Fig. 6, left panel, A-D), large autophagosomes of micrometric size were observed, characterized by a double membrane (arrows in insets) and filled with small, multiple membrane vesicles, other single membrane vesicles, as well as with granular material. At $15 \mathrm{~min}$ post infection (Fig. 6, right panel, E-F), large autophagosomes contained fewer membrane vesicles of different sizes, as well as granular material. At 30 min (Fig. 6, right panel, G-H), autophagosomes appeared almost empty, with residual granular material. Moreover, at both 15 and $30 \mathrm{~min}$, single membrane vacuoles of different size and electron densities and with heterogeneous content were observed, including enlarged lysosomes, multivesicular bodies and autolysosomes (Fig.S2 A-D).

\section{A}

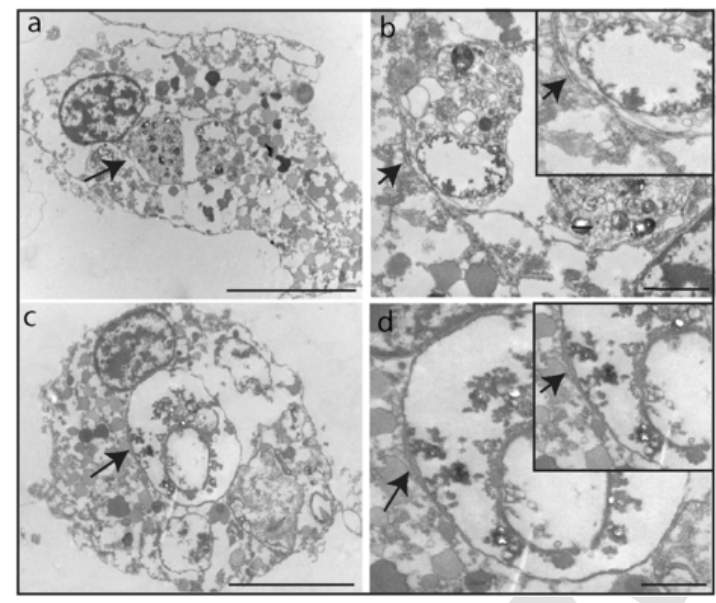

\subsection{Effects of $V$. tapetis on autophagic markers}

The possible effects of $V$. tapetis on expression of autophagic markers was evaluated by electrophoresis and western blotting in hemocytes incubated with $V$. tapetis in the same conditions utilized for TEM analyses. When expression of LC3 was evaluated, the results show that bacterial challenge induced a rapid (from $5 \mathrm{~min}$ ) and progressive increase in the LC3-II/LC3-I ratio (up to two-fold increase with respect to controls at 30 min; $\mathrm{P} \leq 0.05$ ) (Fig. 7A and C); a comparable increase was observed in the LC3-II/actin ratio (not shown).

The mTor pathway is an evolutionarily conserved signaling pathway that senses intra- and extracellular nutrients, growth factors, and pathogen-associated molecular patterns to regulate the function of immune cells [21]. In mussel hemocytes, antibodies directed against the phosphorylated mTor form (p-mTor) recognized a protein band of approximately $290 \mathrm{kDa}$, consistent with the size of mammalian mTor protein (Fig. 7B). V. tapetis induced a time-dependent decrease in the level of p-mTor (about $-50 \%$ and $-75 \%$ with respect to controls, at 15 and 30 min, respectively, $\mathrm{P} \leq 0.05$ ) (Fig. $7 \mathrm{C}$ ). Not significant changes were

B

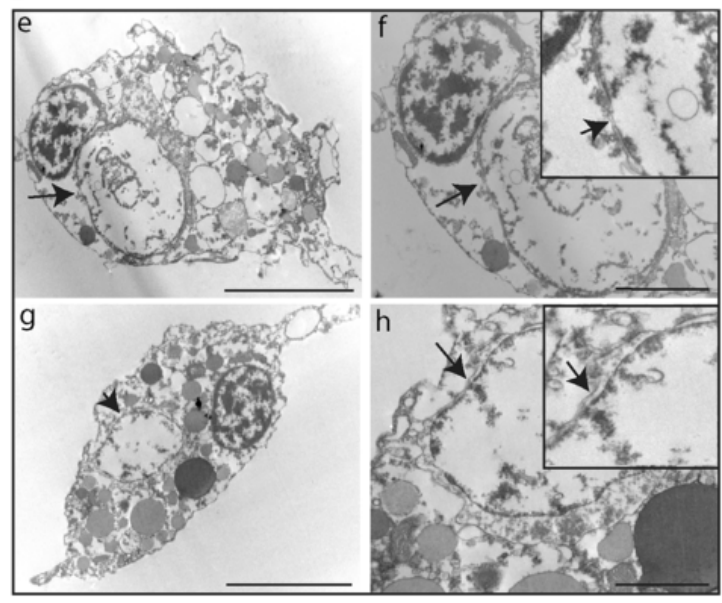

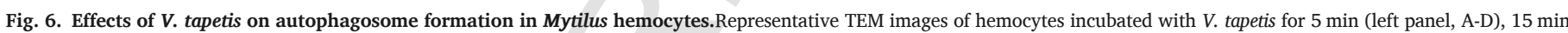

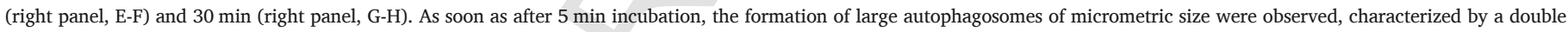

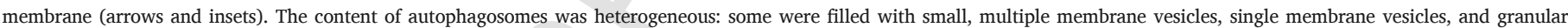

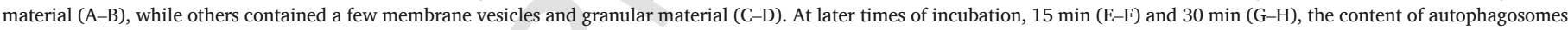
was further decreased to fewer small vesicles and granular material. Scale bars a,c,e,g $=5 \mu \mathrm{m} ; \mathrm{b}, \mathrm{f}, \mathrm{d}, \mathrm{h}=1 \mu \mathrm{m}$.
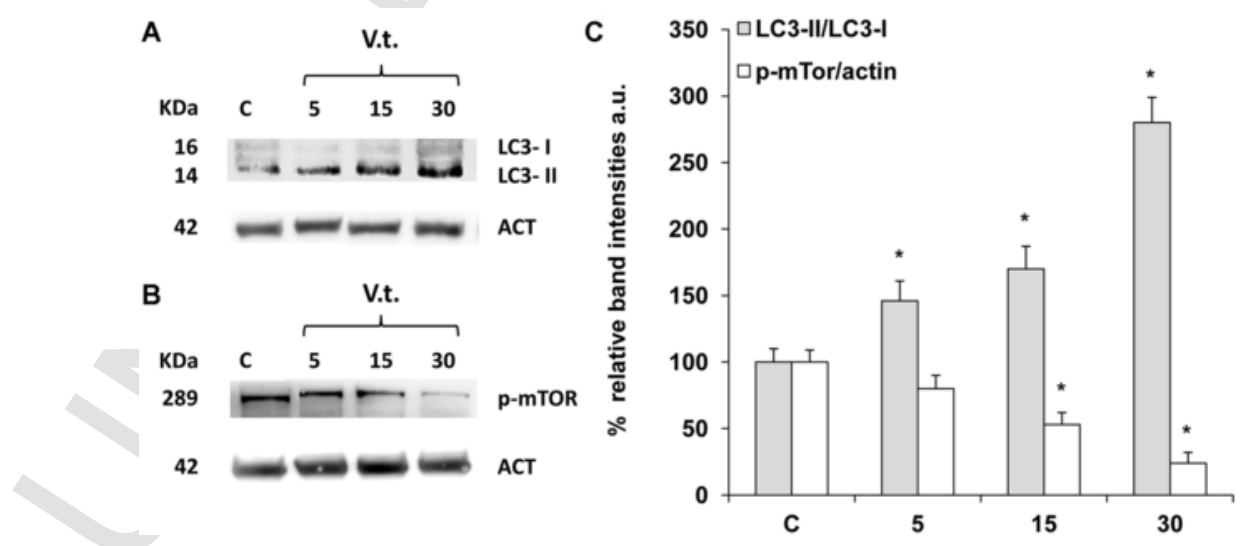

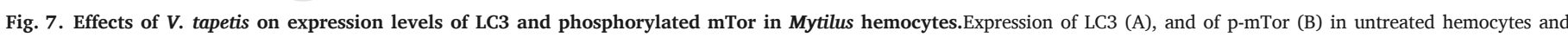

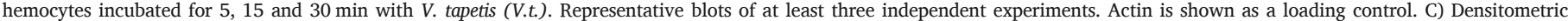
analysis of blots from four independent experiments are presented as mean $\pm \mathrm{SD}$. ${ }^{*} \mathrm{P} \leq 0.05$, Mann-Whitney $U$ test. 
observed in the levels of LC3 and p-mTor in untreated hemocytes from 5 to $30 \mathrm{~min}$ (not shown).

Finally, the level of p62 (SQSTM1/sequestosome 1), an autophagy receptor that links ubiquitinated proteins to LC3, thus serving as a selective substrate of autophagy, was evaluated in hemocytes incubated with $V$. tapetis (5, 15 and $30 \mathrm{~min}$ ) and with Rapamycin for $60 \mathrm{~min}$, and the results are reported in Fig. 8. Due to the longer exposure time to Rapamycin, the time course of p62 expression in control samples is also shown. $V$. tapetis induced a significant decrease in the immunoreactive p62 protein band at $30 \mathrm{~min}$ of incubation (about $-40 \%$ with respect to controls, $\mathrm{P} \leq 0.05$ ) (Fig. 8A-B). A larger, dramatic decrease in the level of p62 was induced by cell exposure to Rapamycin.

\section{Discussion}

The results of the present work represent the first demonstration of autophagic processes in the immune cells of the marine bivalve Mytilus, and of their rapid modulation by bacterial challenge.

In molluscan cells, lysosomal and autophagic reactions have been widely used as indicators of cell injury in response to a wide range of environmental stressors. In Mytilus digestive gland cells, LMS is inversely correlated with efficiency of protein synthesis, autophagic protein turnover, DNA damage, as well as lysosomal swelling, lipidosis and lipofuscinosis [7-9]. In this light, in a first attempt to investigate autophagic pathways in Mytilus immune cells, determination of LMS by the NRRT assay was utilized as a simple, quantitative screening tool for lysosomal reactions in the presence of classical activators/inhibitors of mammalian autophagy. The results showed that Rapamycin, a major negative regulator of autophagy, induced a dose dependent decrease in hemocyte LMS, in the same concentration range utilized in mammalian cells. The effect of Rapamycin was abolished by cell pre-treatment with Wortmannin, that suppresses autophagy via inhibition of PI3-Kinase upstream of mTor [22]. The effect of Rapamycin on the lysosomal sys-

A
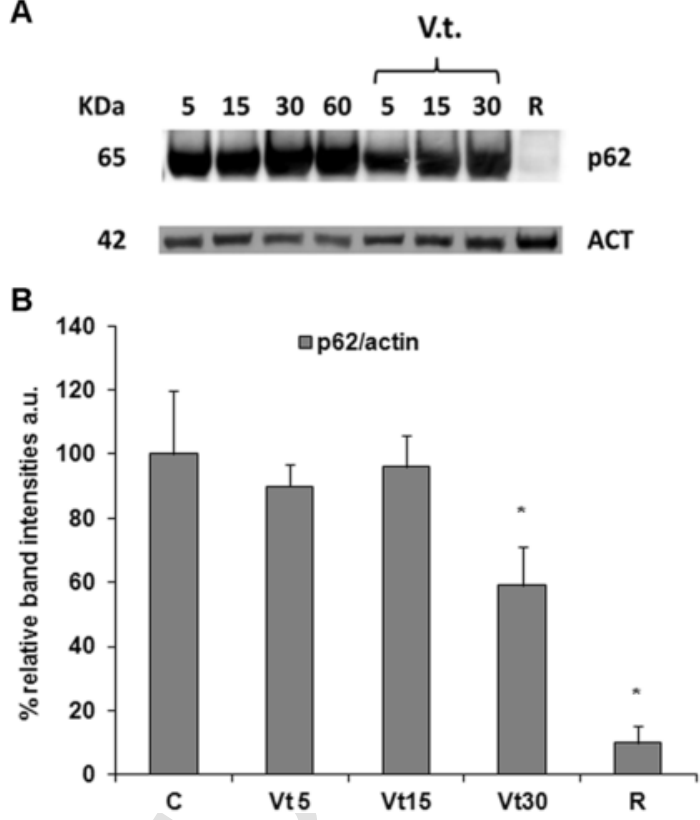

Fig. 8. Expression levels of p62 SQSTM1 in Mytilus hemocytes.A) Expression of p62 in hemocytes incubated for 5, 15 and 30 min with $V$. tapetis (V.t.) and with Rapamycin $(60 \mathrm{~min}, 0.5 \mu \mathrm{M})$. Samples of control hemocytes at all times of incubation are also shown. Actin was used as a loading control. B) Densitometric analysis of blots from four independent experiments. Data, expressed as \% p62/actin ratio of samples incubated with $V . t$. or Rapamycin with respect controls at each time point, are presented as mean \pm SD. ${ }^{*} \mathrm{P} \leq 0.05$, Mann-Whitney $U$ test. tem was confirmed by increased AO fluorescence, evaluated by CLSM. Moreover, Rapamycin induced mitochondrial membrane depolarization in mussel hemocytes; this is in line with previous data demonstrating that pharmacological inhibition of mTor lowered mitochondrial membrane potential, oxygen consumption, and ATP synthetic capacity in mammalian cells [23].

CLSM also showed that Rapamycin increased the signal of the acidotropic dye MDC that, though not specific for early autophagosomes, labels later stages in the degradation process. Although also MDC staining is not, by itself, a sufficient method for monitoring autophagy, it is useful when used in conjunction with other markers [19]. Therefore, the effect of Rapamycin on LC3 expression, which is commonly used as a specific marker of autophagic vacuoles, was evaluated. Increased levels of the activated, lipidated form of LC3, LC3-II, were shown in the hemocytes of crabs injected with Rapamycin and CpG oligodeoxynucleotides [11] and in the mantle tissue of oysters infected with OsHV-1 or treated with $\mathrm{NH}_{4} \mathrm{Cl}$ [10]. The present data show that in vitro treatment with Rapamycin induced a significant increase in LC3-II in mussel hemocytes. Interestingly, the effects were particularly rapid, since they were observed after only $60 \mathrm{~min}$ of incubation, with respect to those observed in mammalian cells (at least $24 \mathrm{~h}$ ). This is in line with the fast response of mussel hemocytes to different types of extracellular stimuli [24].

Autophagy is an important regulator of innate immunity as it helps clear pathogens and control the inflammatory response [25]. Mytilus hemocytes have been shown to represent a suitable invertebrate cell model for investigating the signaling mechanisms involved in the immune response (reviewed in Ref. [13]). These cells are quite resistant to bacterial infection, due to the rapid activation of ROS and NO production, release of hydrolytic enzymes, and phagocytosis, leading to bacterial killing [12]. Bacterial challenge induces lysosomal destabilization, activation of stress activated MAPK (mitogen activated protein kinase), PKC (protein kinase C) and PI-3K, and different immune parameters. All these responses were modulated to a different extent by different bacterial species and strains $[13,14,16]$. In particular, certain bivalve pathogens such as $V$. splendidus LGP32, that did not fully activate the immune response, also lead to lysosomal damage and dysregulation of p38 MAPK signaling [13,15].

$V$. tapetis has been isolated in various mollusks and fish worldwide with about 40 strains identified, with the CECT4600 strain representing the most pathogenic to the Manila clam $V$. philippinarum, where it is considered the causative agent for brown ring disease (BRD) responsible for mass mortalities $[26,27]$. However, little information is available on the effects of $V$. tapetis in mussels. Although the LP2 strain utilized in the present work, isolated from fish (Symphodus melopss) is less cytotoxic to clams with respect to other strains [27], mussel hemocytes were unable to mount an efficient immune response towards this strain. Moreover, $V$. tapetis even reduced the basal ROS production, indicating impairment of the oxidative burst response, and was not killed by hemocytes even at low bacterial concentrations. However, $V$. tapetis induced a moderate lysosomal membrane destabilization $(<50 \%)$, corresponding to activation of the lysosomal machinery in response to mild stress conditions in the absence of cytotoxicity [18]. As for Rapamycin, the vibrio-induced decrease in LMS was prevented by cell pre-treatment with Wortmannin, indicating the involvement of PI3K. Preliminary results indicate $V$. tapetis CECT4600 showed effects comparable with those observed with the LP2 strain on LMS and immune parameters, including the absence of bactrericidal activity (not shown).

In order to clarify whether $V$. tapetis LP2 may affect the autophagic pathways, TEM was utilized as the only tool that reveals the morphology of autophagic structures at a resolution in the nm range [19]. The results show that $V$. tapetis induced the rapid formation of large autophagosomes, characterized by a double membrane, whose content was extremely heterogeneous. The presence of other single membrane 
vacuoles of different sizes and with different contents was also observed; these may represent late/degradative autophagic vacuoles/autolysosomes [19]. Interestingly, none of these structures contained internalized bacteria, indicating that their formation was not related to bacterial degradation, but rather to a process of digestion of damaged cellular components. In fact, few, individual, intact bacteria were observed only within small single membrane vacuoles, probably phagosomes. The lack of signs of bacterial degradation, together with the impairment of the oxidative burst, indicates frustrated phagocytosis, in line with the absence of bactericidal activity.

The effects of other vibrio species and strains associated with oyster mortality outbreaks on Mytilus hemocytes have been previously investigated by TEM in the same experimental conditions [15]. V. aestuarianus 01/032 was rapidly internalized and degraded within the lysosomal system, in line with the efficient bactericidal activity. In contrast, in experiments with $V$. splendidus LGP32, several intact bacteria were observed within large phagosomes; this strain induced early fusion events between lysosomes and was resistant to intracellular killing [15]. However, neither strain induced the formation of autophagosomes, indicating that the effect of $V$. tapetis on autophagy of mussel hemocytes was specific.

In order to investigate the mechanisms involved, biochemical markers of autophagy were evaluated. $V$. tapetis induced a rapid and time dependent increase in the level of LC3-II, consistent with the appearance of autophagosomes observed by TEM. The level of phosphorylated mTor was also evaluated. mTor is a serine/threonine kinase that plays a central role in autophagic signaling complexes and pathways; upon activation, the two mTor complexes (mTORC1 and mTORC2) are phosphorylated on several residues [28]. mTor activity is inhibited under nutrient starvation and other stimuli of autophagy induction, and its dephosphorylation is a crucial step in the induction of autophagy $[1,29]$. The results show that in mussel hemocytes $V$. tapetis induced a time dependent decrease in the immunoreactive band corresponding to p-mTor. Transcripts for mTor have been identified in the tissues Mytilus edulis [30]; moreover, we have recently shown that the level of mRNA for mTor are significantly modulated during early embryo development in M. galloprovincialis [31]. However, in the present work, Western blot with antibodies to total, unphosphorylated mTor showed a high variability of the signal in control hemocytes (not shown); therefore, the level of p-mTor was evaluated by densitometric analysis of the mTor/actin ratio.

An accumulation of autophagosomes, or changes in the amount of LC3-II,are not measures of the autophagic flux per se, but can reflect the induction of autophagic sequestration and/or inhibition of autophagosome or amphisome clearance [19]. In order to gain an insight on the effects of $V$. tapetis on autophagic degradation, expression of the p62 SQSTM1 protein was evaluated. SQSTM1 is an adaptor protein with multiple protein-protein interaction domains, including a LC3 interaction region. p62 and p62 bound to polyubiquitinated proteins are incorporated into the completed autophagosome and are degraded in autolysosomes [19]. The results show that in mussel hemocytes $V$. tapetis induced a significant decrease in the level of p62. Although the limitations of the use of heterologous antibodies directed against epitopes of the mammalian proteins in Western blotting must be taken into account, the observed changes of LC3-II, p-mTor and p62 expression induced by $V$. tapetis are in line with TEM observations. Taken together, the results indicate that $V$. tapetis significantly affects the autophagic pathways in mussel hemocytes. However, from these first data we cannot at present establish whether, in our experimental conditions, hemocyte challenge with $V$. tapetis leads to autophagy induction or the observed autophagy could be the consequence of autophagic flux blockade.

Autophagy participates in the complex mechanisms involved in host-pathogen interactions. Although many bacteria, including vibrios, may utilize autophagy to gain access to essential nutrients, or to exert a general inhibition of vesicle trafficking to avoid phagocytosis [32], autophagy is generally considered to play a protective role in innate immunity, acting as an intracellular surveillance system to monitor and trap invading pathogens (xenophagy) [4,33,34]. However, autophagy does not necessarily imply mechanisms of resistance (increased intracellular disposal and clearance of bacteria). Autophagic processes can also contribute to tolerance mechanisms, that confer protection to the host by minimizing damage caused by the infection [35,36]. In particular, membrane damage and/or pore-forming toxins induce classic starvation responses, by triggering signals dedicated to maintenance of energy and nutrient homeostasis, that may explain recovery of the host cells from sublethal attack [37-41]. In oyster and clam hemocytes, V. tapetis extracellular products (ECPs) induced loss of adherence and inhibition of phagocytosis [42 and references quoted therein]. The recently characterized secretome of $V$. tapetis includes serine proteases, chitinases, and phosholipases, that may manipulate the host signaling pathways [42]: in this light, the role of $V$. tapetis ECPs may affect autophagy in mussel hemocytes deserves further investigation.

Overall, the results obtained in vitro in Mytilus hemocytes suggest that modulation of autophagic pathways induced by certain vibrios (or their products) in immune cells may contribute to tolerance to bacterial infection as a major homeostatic pathway in mussels in vivo. In oysters, autophagy has been shown to play a protective role against $V$. aestuarianus, as seen by survival assay, although no evidence of the involvement of autophagic processes in vibrio elimination was provided [10]. These data support the hypothesis that, in marine invertebrates, autophagy is not necessarily involved in elimination of pathogens, but it can play a protective role against infection $[10,11]$. However, the results of the present work were obtained in vitro, in short exposure experiments, where hemocytes showed little internalization of $V$. tapetis, and no bactericidal activity or induction of oxidative burst were observed. The role of autophagic processes in elimination of $V$. tapetis can be better addressed only in in vivo experiments. In this light, preliminary data obtained in mussels injected with $V$. tapetis indicate that up to $6 \mathrm{~h}$ post injection bacteria persist in mussel hemolymph, and confirm the absence of bactericidal activity and induction of oxidative burst. Moreover, in the hepatopancreas, an increase in lysosomal lipofuscin content, often utilized as an indicator of autophagic processes [7-9], was observed (unpublished results). Overall, these data obtained in vivo would support the observations made in vitro on hemocytes, and suggest that, whatever the mechanisms, the process of clearance of $V$. tapetis from mussels is slow compared with other vibrios [12-14]. Other experiments are under way at longer times of exposure in order to investigate in more detail the involvement of autophagy in the elimination of $V$. tapetis.

With regards to possible changes in gene expression induced by $V$. tapetis in mussel hemocytes, the effects of observed in vitro were too rapid for them to occur. Although the Mytilus genome is not available yet [43] a number of autophagy-related genes are being identified in mussels and oysters, including mTor, different ATG-like genes, Beclin1 and BCL-related genes, and different genes involved in endo-lysosomal trafficking, that are also closely connected to immune-related pathways $[29,44,45]$. The identification of these gene sequences point towards a well developed autophagic machinery in marine bivalves. As previously underlined, in marine invertebrates autophagic processes have mainly been investigated within the context of ecotoxicology, or oxidative stress conditions induced by various environmental stressors [7-9]. In order to clarify the possible involvement of autophagy in mussel tolerance to bacterial infection, studies are in progress to evaluate the effects of in vivo challenge with $V$. tapetis on expression of key autophagy genes both in hemocytes and at the tissue level. These data will contribute to provide further information on the role of autophagic processes in innate immunity of lower organisms. 


\section{Acknowledgments}

This work was funded by the EU project H2020 VIVALDI (Preventing and Mitigating Farmed Bivalve Diseases), contract $n^{\circ} 678589$.

The authors wish to thank Dr. Rita Fabbri and Dr. Michele Montagna for their invaluable technical assistance, and Dr. Chiara Grande and Dr. Giovanni Tassistro for bacterial cultures.

\section{Appendix A. Supplementary data}

Supplementary data related to this article can be found at https:// doi.org/10.1016/j.fsi.2017.12.003.

\section{References}

[1] Z. Yang, D.J. Klionsky, Eaten alive: a history of macroautophagy, Nat. Cell Biol. 12 (2010) 814-822.

[2] C. He, D.J. Klionsky, Regulation mechanisms and signaling pathways of autophagy, Annu. Rev. Genet. 43 (2009) 67-93.

[3] L. Galluzzi, E.H. Baehrecke, A. Ballabio, P. Boya, J.M. Bravo-San Pedro, F. Cecconi, et al., Molecular definitions of autophagy and related processes, Embo J. 36 (2017) 1811-1836.

[4] K. Mao, D.J. Klionsky, Xenophagy: a battlefield between host and microbe, and a possible avenue for cancer treatment, Autophagy 13 (2017) 223-224.

[5] D. Malagoli, F.C. Abdalla, Y. Cao, Q. Feng, K. Fujisaki, A. Gregorc, et al., Autophagy and its physiological relevance in arthropods: current knowledge and perspectives, Autophagy 6 (2010) 575-588.

[6] O. Lamiable, J. Arnold, I.J. de Faria, R.P. Olmo, F. Bergami, C. Meignin, et al., Analysis of the contribution of hemocytes and autophagy to Drosophila antiviral immunity, J. Virol. 90 (2016) 5415-5426.

[7] M.N. Moore, Diet restriction induced autophagy: a protective system against oxidative- and pollutant-stress and cell injury, Mar. Environ. Res. 58 (2004) 603-607.

[8] M.N. Moore, J.I. Allen, A. McVeigh, J. Shaw, Lysosomal and autophagic reactions as diagnostic and predictive indicators of environmental pollutant toxicity in aquatic animals, Autophagy 2 (2006) 217-220.

[9] M.N. Moore, Autophagy as a second level protective process in conferring resistance to environmentally-induced oxidative stress, Autophagy 4 (2008) 254-256.

[10] P. Moreau, K. Moreau, A. Segarra, D. Tourbiez, M.A. Travers, D.C. Rubinsztein, et al., Autophagy plays an important role in protecting Pacific oysters from OsHV-1 and Vibrio aestuarianus infections, Autophagy 11 (2015) 516-526.

[11] M. Sun, L. Wang, S. Jiang, R. Liu, D. Zhao, H. Chen, et al., CpG ODNs induced autophagy via reactive oxygen species (ROS) in Chinese mitten crab, Eriocheir sinensis, Dev. Comp. Immunol. 52 (2015) 1-9.

[12] L. Canesi, G. Gallo, M. Gavioli, C. Pruzzo, Bacteria-hemocyte interactions and phagocytosis in marine bivalves, Microsc. Res. Tech. 57 (2002) 469-476.

[13] L. Canesi, C. Pruzzo, Specificity of innate immunity in bivalves: a lesson from bacteria, in: L. Ballarin, M. Cammarata (Eds.), Lessons in Immunity: from Single-cell Organisms to Mammals, Elsevier Inc, 2016, pp. 79-91.

[14] L. Canesi, M. Betti, C. Ciacci, L.C. Lorusso, G. Gallo, C. Pruzzo, Interactions between Mytilus haemocytes and different strains of Escherichia coli and Vibrio cholerae O1 El Tor: role of kinase-mediated signalling, Cell Microbiol. 7 (2005) 667-674.

[15] T. Balbi, R. Fabbri, K. Cortese, A. Smerilli, C. Ciacci, C. Grande, et al., Interactions between Mytilus galloprovincialis hemocytes and the bivalve pathogens Vibrio aestuarianus 01/032 and Vibrio splendidus LGP32, Fish. Shellfish Immunol. 35 (2013) 1906-1915.

[16] C. Ciacci, A. Manti, B. Canonico, R. Campana, G. Camisassi, W. Baffone, et al., Responses of Mytilus galloprovincialis hemocytes to environmental strains of Vibrio parahaemolyticus, Vibrio alginolyticus, Vibrio vulnificus, Fish. Shellfish Immunol. 65 (2017) 80-87.

[17] H. Towbin, T. Stahelin, J. Gordon, Electrophoretic transfer of protein from polyacrylamide gels to nitrocellulose sheets: procedure and some applications, Proc. Natl. Acad. Sci. U. S. A. 76 (1979) 4350-4354.

[18] OSPAR Commission, Background documents and technical annexes for biological effects monitoring, 239, 2013 www.ospar.org.

[19] D.J. Klionsky, K. Abdelmohsen, A. Abe, M.J. Abedin, H. Abeliovich, A. Acevedo-Arozena, et al., Guidelines for the use and interpretation of assays for monitoring autophagy (3rd edition), Autophagy 12 (2016) 1-222.
[20] R. Chiarelli, M. Agnello, M.C. Roccheri, Sea urchin embryos as a model system for studying autophagy induced by cadmium stress, Autophagy 7 (2011) 1028-1034.

[21] T. Weichhart, M. Hengstschläger, M. Linke, Regulation of innate immune cell function by mTor, Nat. Rev. Immunol. 15 (2015) 599-614.

[22] Y.P. Yang, L.F. Hu, H.F. Zheng, C.J. Mao, W.D. Hu, K.P. Xiong, et al., Application and interpretation of current autophagy inhibitors and activators, Acta Pharmacol. Sin. 34 (2013) 625-635.

[23] S.M. Schieke, D. Phillips, J.P. McCoy Jr., A.M. Aponte, R.F. Shen, R.S. Balaban, et al., The mammalian target of rapamycin (mTor) pathway regulates mitochondrial oxygen consumption and oxidative capacity, J. Biol. Chem. 281 (2006) 27643-27652.

[24] L. Canesi, C. Ciacci, T. Balbi, Invertebrate models for investigating the impact of nanomaterials on innate immunity: the example of the marine mussel Mytilus spp, Curr. Bionanotechnol. 2 (2016) 77-83.

[25] K. Cadwell, Crosstalk between autophagy and inflammatory signalling pathways: balancing defence and homeostasis, Nat. Rev. Immunol. 16 (2016) 661-675.

[26] C. Paillard, K. Korsnes, P. Le Chevalier, C. Le Boulay, L. Harkestad, A.G. Eriksen, et al., Vibrio tapetis-like strain isolated from introduced Manila clams Ruditapes philippinarum showing symptoms of brown ring disease in Norway, Dis. Aquat. Org. 81 (2008) 153-161.

[27] G. Richard, C. Le Bris, F. Guérard, C. Lambert, C. Paillard, Immune responses of phenoloxidase and superoxide dismutase in the manila clam Venerupis philippinarum challenged with Vibrio tapetis-part II: combined effect of temperature and two V. tapetis strains, Fish. Shellfish Immunol. 44 (2015) 79-87.

[28] J. Copp, G. Manning, T. Hunter, TORC-specific phosphorylation of mammalian target of rapamycin (mTor): phospho-Ser2481 is a marker for intact mTor signaling complex 2, Cancer Res. 69 (2009) 1821-1827.

[29] R. Watanabe, L. Wei, J. Huang, mTor signaling, function, novel inhibitors, and therapeutic targets, J. Nucl. Med. 52 (2011) 497-500.

[30] E.E. Philipp, L. Kraemer, F. Melzner, A.J. Poustka, S. Thieme, U. Findeisen, et al., Massively parallel RNA sequencing identifies a complex immune gene repertoire in the lophotrochozoan Mytilus edulis, PLoS One 7 (2012) e33091.

[31] T. Balbi, S. Franzellitti, R. Fabbri, M. Montagna, E. Fabbri, L. Canesi, Impact of bisphenol A (BPA) on early embryo development in the marine mussel Mytilus galloprovincialis: effects on gene transcription, Environ. Pollut. 218 (2016) 996-1004.

[32] D.L. Burdette, M.L. Yarbrough, K. Orth, Not without cause: Vibrio parahaemolyticus induces acute autophagy and cell death, Autophagy 5 (2009) 100-102.

[33] L.C. Gomes, I. Dikic, Autophagy in antimicrobial immunity, Mol. Cell 54 (2014) 224-233.

[34] P. Escoll, M. Rolando, C. Buchrieser, Modulation of host autophagy during bacterial infection: sabotaging host munitions for pathogen nutrition, Front. Immunol. 7 (2016) 81.

[35] C.G. Zou, Y.C. Ma, L.L. Dai, K.Q. Zhang, Autophagy protects C. elegans against necrosis during Pseudomonas aeruginosa infection, Proc. Natl. Acad. Sci. U. S. A. 111 (2014) 12480-12485.

[36] K. Maurer, V.J. Torres, K. Cadwell, Autophagy is a key tolerance mechanism during Staphylococcus aureus infection, Autophagy 11 (2015) 1184-1186.

[37] H.A. Saka, M.G. Gutiérrez, J.L. Bocco, M.I. Colombo, The autophagic pathway: a cell survival strategy against the bacterial pore-forming toxin Vibrio cholerae cytolysin, Autophagy 3 (2007) 363-365.

[38] N. Kloft, C. Neukirch, W. Bobkiewicz, G. Veerachato, T. Busch, G. von Hoven, et al., Pro-autophagic signal induction by bacterial pore-forming toxins, Med. Microbiol. Immunol. 199 (2010) 299-309.

[39] X.G. Guo, T.X. Ji, Y. Xia, Y.Y. Ma, Autophagy protects type II alveolar epithelial cells from Mycobacterium tuberculosis infection, Biochem. Biophys. Res. Commun. 432 (2013) 308-313.

[40] S. Elluri, C. Enow, S. Vdovikova, P.K. Rompikuntal, M. Dongre, S. Carlsson, et al., Outer membrane vesicles mediate transport of biologically active Vibrio cholerae cytolysin (VCC) from V. cholerae strains, PLoS One 9 (2014) e106731.

[41] G. von Hoven, N. Kloft, C. Neukirch, S. Ebinger, W. Bobkiewicz, S. Weis, et al., Modulation of translation and induction of autophagy by bacterial exoproducts, Med. Microbiol. Immunol. 201 (2012) 409-418.

[42] S. Madec, V. Pichereau, A. Jacq, M. Paillard, C. Boisset, F. Guérard, et al., Characterization of the secretomes of two vibrios pathogenic to mollusks, PLoS One 9 (2014) e113097.

[43] M. Murgarella, D. Puiu, B. Novoa, A. Figueras, D. Posada, C. Canchaya, A first insight into the genome of the filter-feeder mussel Mytilus galloprovincialis, PLoS One 11 (2016) e0151561.

[44] M. Gerdol, P. Venier, An updated molecular basis for mussel immunity, Fish. Shellfish Immunol. 46 (2015) 17-38.

[45] J. de Lorgeril, R. Zenagui, R.D. Rosa, D. Piquemal, E. Bachère, Whole transcrip tome profiling of successful immune response to vibrio infections in the oyster Crassostrea gigas by digital gene expression analysis, PLoS One 6 (2011) e23142. 